S. Endo and T. Miyata

Nagoya Math. J.

Vol. 56 (1974), 85-104

\title{
ON A CLASSIFICATION OF THE FUNCTION FIELDS OF ALGEBRAIC TORI
}

\author{
SHIZUO ENDO AND TAKEHIKO MIYATA
}

Let $\Pi$ be a finite group and denote by $M_{\Pi}$ the class of all (finitely generated $Z$-free) $\Pi$-modules. In the previous paper [3] we defined an equivalence relation in $M_{I}$ and constructed the abelian semigroup $T(I I)$ by giving an addition to the set of all equivalence classes in $\boldsymbol{M}_{\Pi}$. The investigation of the semigroup $T(I T)$ seems interesting and important, because this gives a classification of the function fields of algebraic tori defined over a field $k$ which split over a Galois extension of $k$ with group $\Pi$.

The purpose of this paper is to obtain information on the structure of the semigroup $T(I)$.

We will recall the definitions given in [2] and [3]. A $\Pi$-module is called a permutation $\Pi$-module if it can be expressed as a direct sum of $\left\{Z \Pi / \Pi_{i}\right\}$ where each $\Pi_{i}$ is a subgroup of $\Pi$. Further a $\Pi$-module $M$ is called a quasi-permutation $\Pi$-module if there exists an exact sequence $0 \rightarrow M \rightarrow S \rightarrow S^{\prime} \rightarrow 0$ where $S$ and $S^{\prime}$ are permutation $\Pi$-modules. The dual module $\operatorname{Hom}_{Z}(M, Z)$ of a $\Pi$-module $M$ is denoted by $M^{*}$. The augmentation ideal of $Z \Pi$ is denoted by $I_{I I}$ and the dual module $I_{I}^{*}$ of $I_{I}$ is called the Chevalley's module of $I I$ ([1], [2]).

Let $k$ be a field. Let $K$ be a Galois extension of $k$ with group $\cong I I$ and let $M$ be a $\Pi$-module with a $Z$-free basis $\left\{u_{1}, u_{2}, \cdots, u_{n}\right\}$. Define the action on the rational function field $K\left(X_{1}, X_{2}, \ldots, X_{n}\right)$ with $n$ variables $X_{1}, X_{2}, \cdots, X_{n}$ over $K$ by putting, for each $\sigma \in \Pi$ and $1 \leqq i \leqq n, \sigma\left(X_{i}\right)=$ $\prod_{j=1}^{n} X_{j}^{m_{i j}}$ when $\sigma \cdot u_{i}=\sum_{j=1}^{n} m_{i j} u_{j}, m_{i j} \in Z$, and denote by $K(M) K\left(X_{1}, X_{2}\right.$, $\cdots, X_{n}$ ) with this action of $\Pi$. It is well known ([7]) that there is a duality between the category of all algebraic tori defined over $k$ which split over $K$ and the category of all $\Pi$-modules. In fact, if $T$ is an algebraic torus defined over $k$ which splits over $K$, then the character

Received January 17, 1974. 
group $X(T)$ of $T$ can be regarded as a $\Pi$-module, and conversely, if $M$ is a $\Pi$-module, then there is an algebraic torus $T$ defined over $k$ which splits over $K$ such that $X(T) \cong M$ as $\Pi$-modules. It should be noted that the function field of an algebraic torus $T$ defined over $k$ which splits over $K$ can be identified with the invariant subfield $K(X(T))^{I I}$ of $K(X(T)$ ).

For $\Pi$-modules $M$ and $N$ we define a relation $M-\frac{(\varepsilon)}{-} N$ if, for any Galois extension $K$ of $k$ with group $\cong \Pi$, there exist variables $X_{1}, X_{2}, \cdots$, $X_{s}$ and $Y_{1}, Y_{2}, \cdots, Y_{t}$ such that $K(M)^{I}\left(X_{1}, X_{2}, \cdots, X_{s}\right)$ is $k$-isomorphic to to $K(N)^{I}\left(Y_{1}, Y_{2}, \cdots, Y_{t}\right)$. Then this is evidently an equivalence relation in $M_{\Pi}$. Let $T(\Pi)$ be the set of all equivalence classes in $M_{\Pi}$ and denote by $[M]$ the equivalence class containing $M \in M_{\Pi}$. Define an addition in $T(I)$ by $[M]+[N]=[M \oplus N]$ for $M, N \in M_{\Pi}$. This makes $T(\Pi)$ an abelian semigroup with unit element [0]. It is noted that, by the Swan's theorem ([12] and [3], (1.2)), a $\Pi$-module $M$ is a quasi-permutation $\Pi$ module if and only if $[M]=[0]$ (i.e., $M \frac{(z)}{(2)}$. The subgroup of $T(I)$ consisting of all invertible elements in $T(\Pi)$ is denoted by $T^{g}(\Pi)$.

As in [3] denote by $C(Z \Pi)$ the projective class group of $Z \Pi$ and define $C^{q}(Z \Pi)=\{[\mathfrak{U}]-[Z \Pi] \in C(Z \Pi) \mid \mathfrak{A}$ is a quasi-permutation, projective ideal of $Z \Pi\}$. Then $C^{q}(Z \Pi)$ is a subgroup of $C(Z \Pi)$ and the factor group $C(Z \Pi) / C^{q}(Z \Pi)$ can be regarded as a subgroup of $T^{q}(\Pi)$. Let $\Omega_{Z \Pi}$ be a maximal order in $Q I I$ containing $Z \Pi$ and denote by $C\left(\Omega_{Z I I}\right)$ the projective class group of $\Omega_{z \pi}$. It has been shown in [3] and [4] that, for a fairly extensive class of finite groups $\Pi, C(Z \Pi) / C^{q}(Z \Pi) \cong C\left(\Omega_{Z I}\right)$. In such cases, the group $C\left(\Omega_{z_{I}}\right)$ can also be regarded as a subgroup of $T^{g}(I)$. Further denote by $G(Z \Pi)$ the Grothendieck group of $Z \Pi$. Let $B_{G}(Z \Pi)$ be the subgroup of $G(Z \Pi)$ generated by all the images of permutation $\Pi$-modules in $G(Z \Pi)$ and define $S w(\Pi)=G(Z \Pi) / B_{G}(Z \Pi)$. Then there exist natural homomorphisms $\theta_{I}: T^{g}(\Pi) \rightarrow S w(\Pi)$ and $\omega_{I}: C\left(\Omega_{Z I}\right) \rightarrow G(Z \Pi)$.

Our main results in this paper are the following:

[I] The following statements on a finite group $\Pi$ are equivalent:

(1) Every Sylow subgroup of $\Pi$ is cyclic.

(2) $T(\Pi)=T^{g}(\Pi)$, i.e., $T(\Pi)$ is a group.

(3) $\left[I_{\Pi}^{*}\right] \in T^{\theta}(\Pi)$.

[II] The following statements on a finite group $\Pi$ are equivalent:

(1) $\Pi$ is a cyclic group or a direct product of a cyclic group of order $n$ and a group with generators $\rho, \tau$ and relations $\rho^{k}=\tau^{2 d}=I$ and $\tau^{-1} \rho \tau$ 
$=\rho^{-1}$ where $d \geqq 1, k \geqq 3$, and both $n$ and $k$ are odd integers such that $(n, k)=1$.

(2) $I_{I I}^{*}$ is a quasi-permutation $\Pi$-module, i.e., $I_{I I}^{*}-\frac{}{(z)} 0$.

(3) The order of $\left[I_{\Pi}^{*}\right]$ in $T(\Pi)$ is finite.

[III] If $\Pi$ is a finite p-group, then $T^{q}(\Pi)=C(Z \Pi) / C^{q}(Z \Pi)$.

[IV] The following statements on a finite group $\Pi$ are equivalent:

(1) $\Pi$ is (i) a cyclic group, (ii) a dihedral group of order $2 p^{c}$ where $p$ is an odd prime and $c \geqq 1$, (iii) a direct product of a cyclic group of order $q^{f}$ and a dihedral group of order $2 p^{c}$ where $f, c \geqq 1, p$ and $q$ are odd primes and $p$ is a primitive $q^{f-1}(q-1)$-th root of unity modulo $q^{f}$, or (iv) a generalized quaternion group of order $4 p^{c}$ where $p$ is an odd prime congruent to 3 modulo 4 and $c \geqq 1$.

(2) $T(I)=C(Z \Pi) / C^{q}(Z \Pi)=C\left(\Omega_{Z \Pi}\right)$.

(3) $T(\Pi)$ is a finite group.

[V] The following statements on a finite group $\Pi$ are equivalent:

(1) $T(\Pi)=C\left(\Omega_{Z I}\right)$ and $\omega_{I}: C\left(\Omega_{Z I}\right) \rightarrow G(Z I)$ is a monomorphism.

(2) $T(\Pi)=T^{g}(I)$ and $\theta_{\Pi}: T(\Pi) \rightarrow S w(I)$ is an isomorphism.

(3) The dual module of a quasi-permutation $\Pi$-module is always a quasi-permutation $\Pi$-module.

(4) If $0 \rightarrow M^{\prime} \rightarrow M \rightarrow M^{\prime \prime} \rightarrow 0$ is an exact sequence of $\Pi$-modules and any two of $M^{\prime}, M, M^{\prime \prime}$ are quasi-permutation $\Pi$-modules, then the third one is a quasi-permutation $\Pi$-module.

[II] is a supplement to [2], (1.9) and [13], Cor. to Th. 7. [III], [IV] and [V] can be regarded as generalizations of the results in [3], §5.

$\S 1$. Let $\Pi$ be a finite group. Let $M_{\Pi}$ be the class of all $\Pi$-modules, let $\boldsymbol{I}_{I}$ be the class of all projective (left) ideals of $Z \Pi$ and let $\boldsymbol{S}_{\Pi}$ be the class of all permutation $\Pi$-modules. Further define:

$$
\begin{aligned}
& \boldsymbol{H}_{\Pi}=\left\{M \in M_{\Pi} \mid H^{1}\left(\Pi^{\prime}, M\right)=0 \text { for every subgroup } \Pi^{\prime} \text { of } \Pi\right\} ; \\
& \boldsymbol{D}_{\Pi}=\left\{M \in M_{\Pi} \mid M \oplus M^{\prime} \cong S \text { for some } M^{\prime} \in M_{\Pi} \text { and } S \in S_{\Pi}\right\} ; \\
& \boldsymbol{L}_{\Pi}=\left\{M \in M_{I I} \mid M \oplus S \cong \mathfrak{A} \oplus S^{\prime} \text { for some } \mathfrak{A} \in \boldsymbol{I}_{\Pi} \text { and } S, S^{\prime} \in \boldsymbol{S}_{\Pi}\right\} .
\end{aligned}
$$

Then it is easily seen that

$$
\boldsymbol{s}_{\Pi} \subseteq \boldsymbol{L}_{\Pi I} \subseteq \boldsymbol{D}_{I I} \subseteq \boldsymbol{H}_{\Pi} \subseteq \boldsymbol{M}_{\Pi} .
$$


LEMMA 1.1. For every $M \in M_{\Pi}$ there exists an exact sequence

$$
0 \longrightarrow N \longrightarrow S \longrightarrow M \longrightarrow 0
$$

of $I I$-modules with $N \in \boldsymbol{H}_{\Pi}$ and $S \in \boldsymbol{S}_{\Pi}$.

Proof. We can construct an epimorphism $\varphi: S \rightarrow M, S \in S_{\Pi}$ such that, for every subgroup $\Pi^{\prime}$ of $\Pi, \varphi / S^{\Pi^{\prime}}: S^{\Pi^{\prime}} \rightarrow M^{\Pi^{\prime}}$ is an epimorphism. If we put $N=\operatorname{Ker} \varphi$, we have an exact sequence $0 \rightarrow N \rightarrow S \rightarrow M \rightarrow 0$. From this we get the exact sequence

$$
0 \longrightarrow N^{\Pi^{\prime}} \longrightarrow S^{\Pi^{\prime}} \stackrel{\varphi / S^{\Pi^{\prime}}}{\longrightarrow} M^{\Pi^{\prime}} \longrightarrow H^{1}\left(\Pi^{\prime}, N\right) \longrightarrow H^{1}\left(\Pi^{\prime}, S\right) \text {. }
$$

Since $\varphi / S^{\Pi^{\prime}}$ is an epimorphism and $H^{1}\left(\Pi^{\prime}, S\right)=0$, it follows that $H^{1}\left(\Pi^{\prime}, N\right)$ $=0$, and therefore $N \in \boldsymbol{H}_{\Pi I}$.

LEMMA 1.2 ([6]). A $\Pi$-module $M$ is contained in $D_{\Pi}$ if and only if any exact sequence $0 \rightarrow N \rightarrow L \rightarrow M \rightarrow 0$ of $\Pi$-modules with $N \in \boldsymbol{H}_{I I}$ splits.

Proof. The only if part is obvious and the if part follows directly from (1.1).

Let $M, N \in M_{\Pi}$. Define a relation $M \overline{\overline{(\imath)}} N$ if, for every Galois extension $K$ of $k$ with group $\cong \Pi, K(M)^{\Pi}$ is $k$-isomorphic to $K(N)^{\Pi}$. It is evident that if $M \underset{\overline{(z)}}{\rightleftharpoons} N$ then $M \underset{(\text { (r) }}{\rightleftharpoons} N$.

LEMMA 1.3 ([8], [6]). Let $0 \rightarrow N \rightarrow L \rightarrow M \rightarrow 0$ be an exact sequence of $\Pi$-modules with $M \in D_{\Pi}$. Then $L \overline{\overline{(\varepsilon)}} N \oplus M$.

Proof. See [8], (1.2.2) or [6], (1.4).

Lemma 1.4. Let $M$ be a $\Pi$-module. Then $M \in D_{\Pi}$ if and only if $M \in D_{\Pi^{\prime}}$ for every Sylow subgroup $\Pi^{\prime}$ of $\Pi$.

Proof. The only if part is obvious. Suppose that $M \in \boldsymbol{D}_{\Pi^{\prime}}$ for every Sylow subgroup $\Pi^{\prime}$ of $\Pi$. By (1.1) there exists an exact sequence $0 \rightarrow$ $N \rightarrow S \rightarrow M \rightarrow 0$ of $\Pi$-modules with $N \in \boldsymbol{H}_{\Pi}$ and $S \in \boldsymbol{S}_{\Pi}$. According to (1.2) this sequence is $\Pi^{\prime}$-split for every $\Pi^{\prime}$. Then this is also $\Pi$-split, as is well known, and therefore $M \oplus N \cong S$. Thus $M \in D_{\Pi}$.

For $n \geqq 1$ we denote by $\Phi_{n}(X)$ the $n$-th cyclotomic polynomial and by $\zeta_{n}$ the primitive $n$-th root of unity. 
THEOREM 1.5. The following statements on a finite group $\Pi$ are equivalent :

(1) Every Sylow subgroup of $\Pi$ is cyclic.

(2) $H_{\Pi}=D_{\Pi}$.

(3) $T(\Pi)=T^{g}(\Pi)$, i.e., $T(\Pi)$ is a group.

(4) $\left[I_{I I}^{*}\right] \in T^{g}(\Pi)$.

Proof. To show (1) $\Rightarrow(2)$ we may assume by (1.4) that $\Pi$ is a cyclic $p$-group. Let $\Pi=\langle\sigma\rangle$ and $|\Pi|=p^{\ell}$. By induction on $\ell$ we will prove that $\boldsymbol{H}_{\Pi}=\boldsymbol{D}_{\Pi}$. Let $M \in \boldsymbol{H}_{\Pi}$ and put $M^{\prime}=\left\{u \in M \mid \Phi_{p \ell}(\sigma) u=0\right\}$ and $M^{\prime \prime}=$ $M / M^{\prime}$. Then we have an exact sequence $0 \rightarrow M^{\prime} \rightarrow M \rightarrow M^{\prime \prime} \rightarrow 0$ of $\Pi$ modules. For every subgroup $\Pi^{\prime}$ of $\Pi$ we have $H^{2}\left(\Pi^{\prime}, M^{\prime}\right)=\hat{H}^{0}\left(\Pi^{\prime}, M^{\prime}\right)$ $=0$, because $M^{\prime \Pi^{\prime}}=0$. Since $M \in H_{\Pi}$ it follows from this that $M^{\prime \prime} \in \boldsymbol{H}_{\Pi}$. Here $M^{\prime \prime}$ can be regarded as a $\Pi /\left\langle\sigma^{p^{\ell-1}}\right\rangle$-module. Therefore by induction we have $M^{\prime \prime} \in D_{\Pi /\left\langle\sigma^{p} p^{\ell-1}\right\rangle} \subseteq D_{I}$, hence $\boldsymbol{M}^{\prime \prime} \oplus N \cong S$ for some $N \in M_{\Pi /\left\langle\sigma^{\left.p^{\ell-1}\right\rangle}\right.}$ and $S \in S_{\Pi /\left\langle p^{\ell-1\rangle}\right.}$. Thus we get an exact sequence $0 \rightarrow M^{\prime} \rightarrow M \oplus N \rightarrow S$ $\rightarrow 0$. On the other hand, $M^{\prime}$ can be considered as a $Z\left[\zeta_{p}\right]-$ module. Since $\Pi$ is a cyclic $p$-group, we can find an exact sequence $0 \rightarrow M^{\prime} \rightarrow T \rightarrow$ $T^{\prime} \oplus \mathfrak{A} \rightarrow 0$ of $\Pi$-modules where $T, T^{\prime} \in S_{\Pi}$ and $\mathfrak{A} \in I_{\Pi}$. Forming the pushout of $M^{\prime} \rightarrow M \oplus N$ we get the exact sequence $0 \rightarrow M \oplus N \rightarrow T \oplus S \rightarrow$ $\downarrow$

$T^{\prime} \oplus \mathfrak{A} \rightarrow 0$. Since $M \oplus N \in \boldsymbol{H}_{\Pi}$, this sequence splits, hence $M \oplus N \oplus \mathfrak{A} \oplus T^{\prime}$ $\cong T \oplus S$. Consequently we have $M \in D_{I}$, which proves that $H_{\Pi}=D_{\Pi}$. Next suppose that $\boldsymbol{H}_{\Pi}=\boldsymbol{D}_{\Pi}$. Let $M \in \boldsymbol{M}_{\Pi}$. Then by (1.1) there exists an exact sequence $0 \rightarrow L \rightarrow S \rightarrow M^{*} \rightarrow 0$ of $\Pi$-modules with $L \in \boldsymbol{H}_{\Pi}$ and $S \in S_{I I}$. Dualizing this we get an exact sequence $0 \rightarrow M \rightarrow S \rightarrow L^{*} \rightarrow 0$. By assumption we have $L \in D_{I}$ and so $L^{*} \in D_{I I}$. Hence we have, by (1.3), $M \oplus L^{*}-0$, which shows that $[M] \in T^{g}(\Pi)$. Thus $T(\Pi)=T^{g}(\Pi)$. This proves $(2) \Rightarrow(3)$. The implication $(3) \Rightarrow(4)$ is obvious. Finally suppose that $\left[I_{I I}^{*}\right] \in T^{g}(\Pi)$. Then $I_{I I}^{*} \oplus N \frac{(z)}{-0}$ for some $N \in M_{\Pi}$. Therefore there exists an exact sequence $0 \rightarrow S^{\prime} \rightarrow S \rightarrow I_{\Pi} \oplus N^{*} \rightarrow 0$ with $S, S^{\prime} \in S_{\Pi}$. From this we get the exact sequence

$$
0 \longrightarrow H^{1}\left(\Pi, I_{\Pi}\right) \oplus H^{1}\left(\Pi, N^{*}\right) \longrightarrow H^{2}\left(\Pi, S^{\prime}\right) .
$$

However $H^{1}\left(\Pi, I_{\Pi}\right) \cong Z /|\Pi| Z$. Therefore $H^{2}\left(\Pi, S^{\prime}\right)$ contains an element of order $|\Pi|$. Then we easily see that every Sylow subgroup of $\Pi$ is cyclic, which completes the proof of $(4) \Rightarrow(1)$. 
We return to the general situation.

LEMMA 1.6. Let $M$ be a $\Pi$-module such that $[M] \in T^{g}(\Pi)$. Then there exists $L \in D_{\Pi}$ such that $L \frac{-}{(r)} M$.

Proof. There is a $\Pi$-module $M^{\prime}$ such that $M \oplus M^{\prime} \frac{}{{ }^{(r)}} 0$. By virtue of (1.1) there exist exact sequences

$$
\begin{aligned}
& 0 \longrightarrow M \longrightarrow S \longrightarrow N \longrightarrow 0 \\
& 0 \longrightarrow M^{\prime} \longrightarrow S^{\prime} \longrightarrow N^{\prime} \longrightarrow 0
\end{aligned}
$$

of $\Pi$-modules where $S, S^{\prime} \in \boldsymbol{S}_{\Pi}$ and $N^{*}, N^{*} \in \boldsymbol{H}_{\Pi}$. Since $M \oplus M^{\prime} \frac{(\text { (z) }}{0 \text {, }}$ we also have an exact sequence $0 \rightarrow M \oplus M^{\prime} \rightarrow T \rightarrow T^{\prime} \rightarrow 0$ with $T, T^{\prime} \in S_{\Pi}$. Forming the pushout of $M \oplus M^{\prime} \rightarrow S \oplus S^{\prime}$, we get the exact sequence

$$
\begin{gathered}
\downarrow \\
0 \longrightarrow T \longrightarrow S \oplus S^{\prime} \oplus T^{\prime} \longrightarrow N \oplus N^{\prime} \longrightarrow 0 .
\end{gathered}
$$

Because $N^{*}, N^{*} \in \boldsymbol{H}_{\Pi}$, we have $N \oplus N^{\prime} \oplus T \cong S \oplus S^{\prime} \oplus T$ and so $N, N^{\prime} \in D_{\Pi}$. From this we get the exact sequence

$$
0 \longrightarrow M \longrightarrow S \oplus N^{\prime} \oplus T \longrightarrow S \oplus S^{\prime} \oplus T^{\prime} \longrightarrow 0 \text {. }
$$

Then from (1.3) it follows that $M \frac{(z)}{{ }^{\prime}} N^{\prime}$. Thus $L=N^{\prime}$ is as desired.

Proposition 1.7. Let $\Pi$ be a finite group. Then the group $T^{g}(\Pi)$ is finitely generated.

Proof. By (1.6) each element of $T^{g}(\Pi)$ has a representative in $\boldsymbol{D}_{\Pi}$. Then, according to [5], (5.8), $T^{g}(I)$ is finitely generated.

The authors do not know whether, for any finite group $\Pi$, the semigroup $T(\Pi)$ is finitely generated or not.

§ 2. In this section we will study the Chevalley's module $I_{I I}^{*}$ more precisely. The torsion part of an abelian group $A$ will be denoted by $t(A)$.

LEMMA 2.1. Let II be a finite group such that $\left(I_{I I}^{*}\right)^{(n)} \frac{}{{ }^{(2)}} 0$ for some $n>0$. Then:

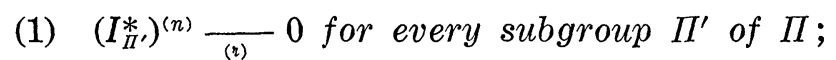

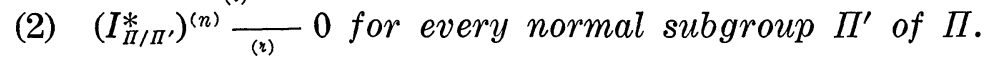

Proof. Let $\Pi^{\prime}$ be a subgroup of $\Pi$ with $\left[\Pi: \Pi^{\prime}\right]=m$. Then it is 
easily seen that $I_{I} \cong I_{\Pi^{\prime}} \oplus Z \Pi^{\prime(m-1)}$ as $\Pi^{\prime}$-modules and therefore we have $I_{I I}^{*} \cong I_{I^{\prime}}^{*} \oplus Z \Pi^{\prime(m-1)}$. This shows that $\left(I_{I^{\prime}}^{*}\right)^{(n)} \frac{{ }^{(z)}}{} 0$. Next suppose that $\Pi^{\prime}$ is a normal subgroup of $\Pi$ with $\left[\Pi: \Pi^{\prime}\right]=m$. Then we have $I_{\Pi / \Pi^{\prime}}^{*}$ $\cong Z \Pi \cdot / \Pi^{\prime} /\left(\sum_{\sigma \Pi^{\prime} \in \Pi / \Pi^{\prime}} \sigma \Pi^{\prime}\right)$ and $Z \Pi / I \Pi^{\prime} \otimes_{Z \Pi} I_{I I}^{*} \cong Z \Pi / \Pi^{\prime} /\left(m \sum_{\sigma \Pi^{\prime} \in \Pi / \Pi^{\prime}} \sigma \Pi^{\prime}\right)$. Therefore $Z \Pi / \Pi^{\prime} \otimes_{Z I I} I_{I I}^{*} / t\left(Z \Pi / \Pi^{\prime} \otimes_{Z I I} I_{I I}^{*}\right) \cong I_{I I / \Pi^{\prime}}^{*}$. Since $\left(I_{I I}^{*}\right)^{(n)}-\frac{}{{ }^{(z)}} 0$, there exists an exact sequence $0 \rightarrow\left(I_{\Pi}^{*}\right)^{(n)} \rightarrow S \rightarrow S^{\prime} \rightarrow 0$ with $S, S^{\prime} \in S_{\Pi}$. Tensoring this with $Z \Pi / \Pi^{\prime}$ over $Z \Pi$, we get the exact sequence

$$
\left(Z \Pi / \Pi^{\prime} \otimes_{Z \Pi} I_{I}^{*}\right)^{(n)} \stackrel{\varphi}{\longrightarrow} Z \Pi / \Pi^{\prime} \bigotimes_{Z \Pi} S \longrightarrow Z \Pi / \Pi^{\prime} \underset{Z \Pi}{\otimes} S^{\prime} \longrightarrow 0 .
$$

Then $\operatorname{Im} \varphi \cong\left(Z \Pi / \Pi^{\prime} \otimes_{Z \Pi} I_{I}^{*} / t\left(Z \Pi / \Pi^{\prime} \otimes_{Z I} I_{I}^{*}\right)\right)^{(n)} \cong\left(I_{I / \Pi^{\prime}}^{*}\right)^{(n)}$. This shows that $\left(I_{I / \Pi^{\prime}}^{*}\right)^{(n)} \frac{}{(z)} 0$.

LEMMA 2.2. Let $\Pi$ be a finite group whose Sylow subgroups are cyclic. Let $0 \rightarrow N \rightarrow L \rightarrow M \rightarrow 0$ be an exact sequence of $I$-modules and suppose that $\hat{H}^{0}\left(\Pi^{\prime}, N\right)=0$ for every subgroup $\Pi^{\prime}$ of $\Pi$. Then $L-\frac{}{(n)}$ $M \oplus N$.

Proof. We can construct an exact sequence $0 \rightarrow N \rightarrow F \rightarrow V \rightarrow 0$ of $\Pi$-modules where $F$ is $Z \Pi$-free. Since every Sylow subgroup of $\Pi$ is cyclic, we have $V \in \boldsymbol{H}_{\Pi}$ and therefore (1.5) shows that $V \in \boldsymbol{D}_{\Pi}$. Forming the pushout of $N \rightarrow L$, we get the exact sequence

$$
\stackrel{\downarrow}{F} \text { }
$$

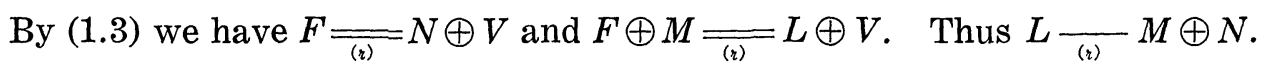

Let $\Pi$ be a finite group whose Sylow subgroups are cyclic. Then $\Pi$ is expressible as a semidirect product of a cyclic normal subgroup $\langle\sigma\rangle$ of order $\ell$ and a cyclic subgroup $\langle\tau\rangle$ of order $m$ such that $(\ell, m)=1$ and for every prime $q \mid m$ a $q$-Sylow subgroup of $\Pi$ is not contained in the centralizer $C(\sigma)$ of $\sigma$. Let $\ell=\ell(\Pi)$ and $m=m(\Pi)$ and define $i(I I)=|\operatorname{Im}(\langle\tau\rangle \rightarrow \operatorname{Aut}\langle\sigma\rangle)|$.

THEOREM 2.3. The following statements on a finite group $I$ are equivalent:

(1) II is a cyclic group or a direct product of a cyclic group of order $n$ and a group with generators $\rho, \tau$ and relations $\rho^{k}=\tau^{2^{d}}=I$ and $\tau^{-1} \rho \tau=\rho^{-1}$ 
where $d \geqq 1, k \geqq 3$ and both $n$ and $k$ are odd integers such that $(n, k)$ $=1$.

(2) $I_{I I}^{*}$ is a quasi-permutation $\Pi$-module, i.e., $I_{I I}^{*}-0$.

(3) The order of $\left[I_{\Pi}^{*}\right]$ in $T(\Pi)$ is finite.

Proof. By (1.5) we may assume that $\Pi$ is a finite group whose Sylow subgroups are cyclic. It should be remarked that the statement (1) is equivalent to the following one:

$\left(1^{\prime}\right) \quad i(\Pi)=1$ or 2 .

The implication $(2) \Rightarrow(3)$ is evident. Hence we only need to prove (3) $\Rightarrow(1)$ and (1) $\Rightarrow(2)$.

First suppose that $i(\Pi)=m(\Pi)=2,4$ or $q$ and $\ell(\Pi)=p^{c}, \quad c \geqq 1$ where $p$ and $q$ are distinct odd primes. Let $\Lambda=Z \Pi /\left(\Phi_{p c}(\sigma)\right) \cong Z\langle\tau\rangle\left[\zeta_{p c}\right]$. Then $\Lambda$ is isomorphic to the trivial crossed product of $Z\left[\zeta_{p c}\right]$ and $\langle\tau\rangle$ and so it is a hereditary order in $Q \Pi /\left(\Phi_{p c}(\sigma)\right)$. Further let $R=Z\left[\zeta_{p c}\right]$ and $\mathfrak{P}=$ $\left(\zeta_{p c}-1\right)$. Then $\mathfrak{B}$ is a prime ideal of $R$ and both $R$ and $\mathfrak{B}$ can be regarded as $\Lambda$-modules. According to a result in [9] we have $\Lambda \cong R \oplus \mathfrak{B} \oplus \cdots \oplus \mathfrak{P}^{i(\pi)-1}$ as $\Lambda$-modules. It is easily seen that all of $\Lambda, \Lambda^{*}, R$ and $R^{*}$ are quasipermutation $\Pi$-modules. Therefore, when $i(\Pi)=2$, we have $\mathfrak{B} \frac{}{(r)} 0$

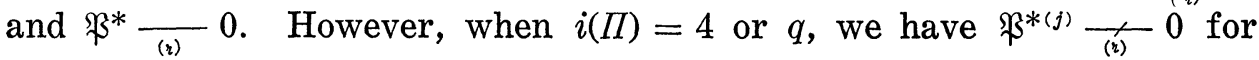
any $j>0$. In fact, if $\mathfrak{P}^{*(j)}-\frac{1(\tau)}{(\eta)}$, then there is an exact sequence $0 \rightarrow$ $S^{\prime} \rightarrow S \rightarrow \mathfrak{P}^{(j)} \rightarrow 0$ with $S^{\prime}, S \in S_{\Pi}$. Tensoring this with $\Lambda$ over $Z \Pi$ and eliminating the torsion parts, we get

$$
\Lambda^{(u)} \oplus R^{(v)} \oplus \mathfrak{P}^{2(w)} \oplus \mathfrak{P}^{(j)} \cong \Lambda^{\left(u^{\prime}\right)} \oplus R^{\left(v^{\prime}\right)} \oplus \mathfrak{P}^{2\left(w^{\prime}\right)} \quad \text { when } i(\Pi)=4
$$

and

$$
\Lambda^{(u)} \oplus R^{(v)} \oplus \mathfrak{P}^{(j)} \cong \Lambda^{\left(u^{\prime}\right)} \oplus R^{\left(v^{\prime}\right)} \quad \text { when } i(\Pi)=q,
$$

where $u, v, w, u^{\prime}, v^{\prime}, w^{\prime}$ are non negative integers. This contradicts the Rosen's result ([9]). Now the $\Pi$-module $\Phi_{p c}(\sigma) I_{I}$ has a free basis

$$
\left\{\Phi_{p c}(\sigma)\left(\sigma^{\alpha}-1\right), \Phi_{p c}(\sigma)\left(\sigma^{\alpha} \tau^{\beta}-1\right), \Phi_{p c}(\sigma)\left(\tau^{\beta}-1\right)\right\}_{0<\alpha<p^{c-1}, 0<\beta<m(I)}
$$

and $\Phi_{p c}(\sigma) I_{I I} \cong I_{I /\left\langle p^{c-1}\right\rangle}$ as $I I$-modules. Therefore $\Lambda \otimes_{Z I I} I_{I I}$ is torsion-free and we have an exact sequence

$$
0 \longrightarrow I_{\Pi /\left\langle p^{p} p^{c-1}\right\rangle} \longrightarrow I_{I I} \longrightarrow \Lambda \underset{Z \Pi}{\bigotimes_{I I}} I_{\Pi} \longrightarrow 0 \text {. }
$$

It is easily seen that $\hat{H}^{0}\left(\Pi^{\prime}, \Lambda\right)=0$ for every subgroup $\Pi^{\prime}$ of $\Pi$. Since 
$\Lambda$ is hereditary, we have $\hat{H}^{0}\left(\Pi^{\prime},\left(\Lambda \otimes_{Z \Pi} I_{\Pi}\right)^{*}\right)=0$ for every subgroup $\Pi^{\prime}$ of $\Pi$. Then, according to (2.2), we have $I_{I I}^{*}-I_{I I /\left\langle p^{p c-1}\right\rangle}^{*} \oplus\left(\Lambda \otimes_{Z I} I_{I I}\right)^{*}$. Tensoring the exact sequence $0 \rightarrow I_{I} \rightarrow Z \Pi \rightarrow Z \rightarrow 0$ with $\Lambda$ over $Z \Pi$, we get the exact sequence

$$
0 \longrightarrow \Lambda \underset{Z \Pi}{\otimes} I_{\Pi} \longrightarrow \Lambda \longrightarrow R / \Re \longrightarrow 0 .
$$

Forming further the pullback of $\Lambda \rightarrow R / \Re$, we see that $\left(\Lambda \otimes_{Z I I} I_{I}\right) \oplus R$ $\stackrel{\uparrow}{R}$

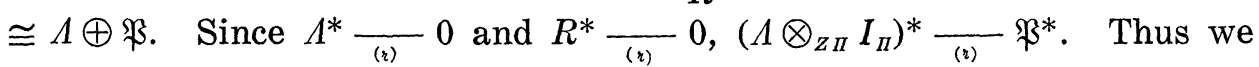
have

$$
I_{I I}^{*} \frac{(\mathrm{z})}{I_{I /\left\langle\sigma p^{c-1}\right\rangle}^{*}} \oplus \mathfrak{B}^{*} \ldots \ldots \ldots \text { (a) . }
$$

(3) $\Rightarrow(1)$ : Suppose that $i(I)>2$. To show that $I I$ does not satisfy (3) we may suppose by (2.1) that $i(I)=m(I)=4$ or $q$ and $\ell(I)=p$ where $p$ and $q$ are distinct odd primes. In this case the group $\Pi /\langle\sigma\rangle$ is cyclic and so $I_{I /\langle\sigma\rangle}^{*} \cong I_{\pi /\langle\sigma\rangle} \overline{(z)} 0$. By virtue of (a) we have $I_{I}^{*} \overline{(z)} \mathfrak{B}^{*}$. However $\mathfrak{B}^{*(j)} \frac{-}{(k)} 0$ for any $j>0$. Therefore the order of $\left[I_{I I}^{*}\right]$ in $T(I)$ is not finite. This completes the proof of $(3) \Rightarrow(1)$.

(1) $\Rightarrow(2)$ : Suppose that $i(\Pi)=1$ or 2 . If $i(I)=1$, then $I_{I I}^{*} \cong I_{I}$ -0 . Hence we suppose that $i(\Pi)=2$. Let $\sigma^{\prime}$ be the generator of a cyclic group of order $n$ and let $\mu=\sigma^{\prime} \rho \tau^{2}$. Then $\langle\mu\rangle$ is the normal subgroup of $\Pi$ of order $b=n k 2^{d-1}$. Let $p$ be a prime divisor of $k$. Let $k=p^{c} k^{\prime},\left(p, k^{\prime}\right)=1$, and let $b^{\prime}=b / p^{c}=n k^{\prime} 2^{d-1}$. Further let $F(X)=$ $\prod_{r \mid b^{\prime}}, \Phi_{p c r}(X)$ and $G(X)=F(X) / \Phi_{p c}(X)$, and let $\Gamma=Z \Pi /(F(\mu)), \quad \Gamma_{0}=$ $Z \Pi /(G(\mu))$ and $\Gamma_{1}=Z \Pi /\left(\Phi_{p c}(\mu)\right)$. Since any of $F(1), G(1)$ and $\Phi_{p c}(1)$ is not 0 , we see that $t\left(\Gamma \otimes_{z I I} I_{I I}\right)=t\left(\Gamma_{0} \otimes_{z I I} I_{I I}\right)=t\left(\Gamma_{1} \otimes_{z I} I_{I I}\right)=0$. Hence, tensoring the exact sequence $0 \rightarrow \Gamma_{1} \rightarrow \Gamma \rightarrow \Gamma_{0} \rightarrow 0$ with $I_{\Pi}$ over $Z \Pi$, we get the exact sequence

$$
0 \longrightarrow \Gamma_{1} \underset{Z I I}{\otimes} I_{I I} \longrightarrow \Gamma \underset{Z I}{\otimes} I_{I} \longrightarrow \Gamma_{0} \underset{Z I I}{\otimes} I_{I} \longrightarrow 0
$$

Because $G(1)= \pm 1$, we can show that $\Gamma_{0} \otimes_{Z I} Z=0$. Therefore, tensoring the exact sequence $0 \rightarrow I_{\Pi} \rightarrow Z \Pi \rightarrow Z \rightarrow 0$ with $\Gamma_{0}$ over $Z \Pi$, we see that $\Gamma_{0} \otimes_{Z I} I_{I I} \cong \Gamma_{0}$. Let $H(X)=X^{b}-1 / G(X)$ and $\Gamma^{\prime}=Z \Pi /(H(\mu))$. Then there is an exact sequence $0 \rightarrow \Gamma^{\prime} \rightarrow Z \Pi \rightarrow \Gamma_{0} \rightarrow 0$. Forming the pullback of $\Gamma \otimes_{z I} I_{I} \rightarrow \Gamma_{0}$, we get the exact sequence $\uparrow$ 


$$
0 \longrightarrow \Gamma^{\prime} \longrightarrow\left(\Gamma_{1} \otimes_{Z I} I_{I I}\right) \oplus Z I \longrightarrow \longrightarrow \Gamma \otimes_{Z \Pi} I_{I I} \longrightarrow 0 \cdots(\mathrm{b})
$$

The group $\Pi /\left\langle\mu^{p^{c}}\right\rangle$ is dihedral and of order $2 p^{c}$ and there is a natural epimorphism $Z \Pi /\left\langle\mu^{p^{c}}\right\rangle \rightarrow \Gamma_{1}$. It is seen that $\Gamma_{1} \otimes_{Z \Pi} I_{I I} \cong \Gamma_{1} \otimes_{Z \Pi /\langle\mu p c} I_{\Pi /\left\langle\mu p^{c}\right\rangle}$. Therefore, as shown in the proof of (a), $\left(\Gamma_{1} \otimes_{Z I I} I_{I I}\right)^{*}-\frac{}{(r)} 0$. On the other hand, there exists the following commutative diagram with exact rows and columns :

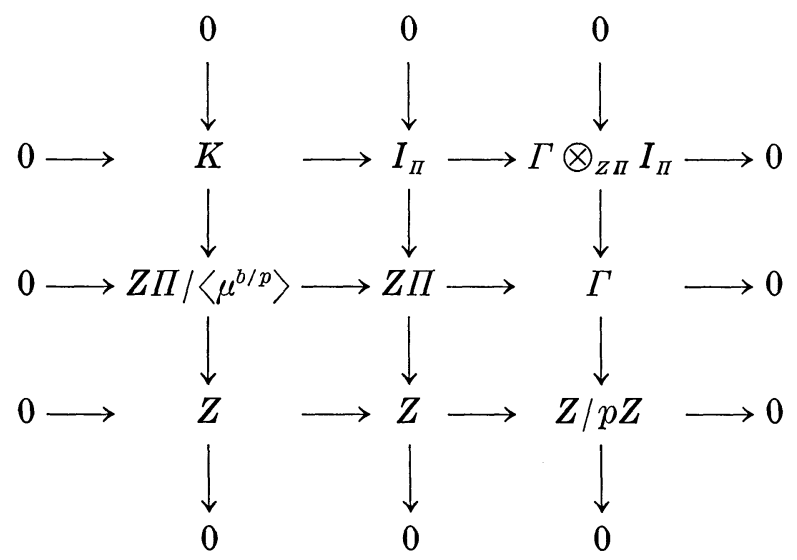

Then $K \cong I_{\pi /\langle\mu b / p\rangle}$. Thus we have the exact sequence

$$
0 \longrightarrow I_{I I / \mu^{b / p\rangle}} \longrightarrow I_{I I} \longrightarrow \Gamma \bigotimes_{Z I I} I_{I I} \longrightarrow 0 \cdots \cdots(\mathrm{c}) \text {. }
$$

Now it is easily shown that $\hat{H}^{0}\left(\Pi^{\prime}, \Gamma\right)=0$ for every subgroup $\Pi^{\prime}$ of $\Pi$. From the exact sequence $0 \rightarrow \Gamma \otimes I_{I I} \rightarrow \Gamma \rightarrow Z / p Z \rightarrow 0$ we get the exact sequence

$$
\hat{H}^{-1}\left(\Pi^{\prime}, \Gamma\right) \stackrel{\varphi}{\longrightarrow} \hat{H}^{-1}\left(\Pi^{\prime}, Z / p Z\right) \longrightarrow \hat{H}^{0}\left(\Pi^{\prime}, \Gamma \underset{Z \Pi}{\otimes} I_{\Pi}\right) \longrightarrow 0 .
$$

By a direct computation we can show that $\varphi$ is an epimorphism. Hence, for every subgroup $\Pi^{\prime}$ of $\Pi, \hat{H}^{0}\left(\Pi^{\prime}, \Gamma \otimes_{z I I} I_{I I}\right)=0$, and so $\hat{H}^{0}\left(\Pi^{\prime},\left(\Gamma \otimes_{z I I} I_{I I}\right)^{*}\right)$ $=0$. From (2.2) and (b) it follows that $\left(\Gamma \otimes_{z \Pi} I_{I I}\right)^{*}-0$, because $\Gamma^{*}$ (z) 0 and $\left(\Gamma_{1} \otimes_{Z I} I_{I I}\right)^{*}-0$. Furthermore, applying (2.2) to (c), we

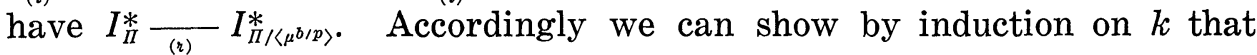

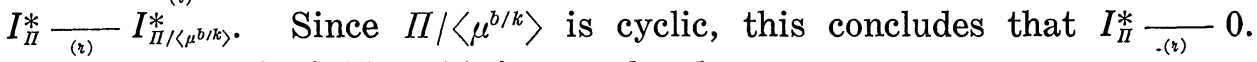
Thus the proof of $(1) \Rightarrow(2)$ is completed. 


\section{§3. We first give}

Proposition 3.1. The following statements on a finite group $\Pi$ are equivalent :

(1) $D_{\Pi}=L_{\Pi I}$

(2) $T^{g}(\Pi)=C(Z \Pi) / C^{q}(Z \Pi)$.

Proof. This is an immediate consequence of (1.6).

THEOREM 3.2. Let $\Pi$ be a finite $p$-group. Then $T^{q}(\Pi)=C(Z \Pi) / C^{q}(Z \Pi)$.

Proof. By (3.1) it suffices to show that $D_{\Pi}=L_{\Pi}$. If $M \in D_{I}$, there are $M^{\prime} \in D_{I}$ and $S \in S_{I}$ such that $M \oplus M^{\prime} \cong S$. Since $\Pi$ is a $p$-group, for every subgroup $\Pi^{\prime}$ of $\Pi, Z_{p} \Pi / \Pi^{\prime}$ is an indecomposable $Z_{p} \Pi$-module. It is well known that the Krull-Schmidt theorem holds for finitely generated $Z_{p} \Pi$-modules. Hence there exists $T \in S_{I}$ such that $T_{p} \cong M_{p}$ as $Z_{p} \Pi$ modules. This implies that $T$ and $M$ have the same genus. Then there is $\mathfrak{U} \in I_{I}$ such that $M \oplus Z \Pi \cong T \oplus \mathfrak{U}$. This proves that $M \in L_{\Pi}$.

We did not succeed in determining all finite groups $\Pi$ such that $T^{g}(\Pi)=C(Z \Pi) / C^{q}(Z \Pi)$. It is shown that a finite nilpotent group $\Pi$ is a $p$-group if and only if, for any $M \in M_{I I}$ such that $M \oplus S^{\prime} \cong S$ for some $S^{\prime}, S \in S_{\Pi}$, there is $T \in S_{\Pi}$ whose genus is the same as that of $M$. Hence our method used in the proof of (3.2) can not be applied to nilpotent groups.

However we can determine all finite groups $\Pi$ such that $T(\Pi)=$ $C(Z \Pi) / C^{q}(Z \Pi)$.

THEOREM 3.3. The following statements on a finite group $I I$ are equivalent:

(1) $I I$ is (i) a cyclic group, (ii) a dihedral group of order $2 p^{c}$ where $p$ is an odd prime and $c \geqq 1$, (iii) a direct product of a cyclic group of order $q^{f}$ and a dihedral group of order $2 p^{c}$ where $f, c \geqq 1, p$ and $q$ are odd primes and $p$ is a primitive $q^{f-1}(q-1)$-th root of unity modulo $q^{f}$, or (iv) a generalized quaternion group of order $4 p^{c}$ where $c \geqq 1$ and $p$ is an odd prime congruent to 3 modulo 4.

(2) $T(\Pi)=C(Z \Pi) / C^{q}(Z \Pi)=C\left(\Omega_{Z \Pi}\right)$.

(3) $T(\Pi)$ is a finite group.

Proof. It can easily be seen that the statement (1) is equivalent to the following one: 
(1') $\Pi$ is (i') a cyclic group or (ii') a direct product of a cyclic group of order $n$ and a group with generators $\rho, \tau$ and relations $\rho^{p^{c}}=\tau^{2^{d}}=I$ and $\tau^{-1} \rho \tau=\rho^{-1}$ where $c, d \geqq 1, p$ is an odd prime which is a prime in $Z\left[\zeta_{n_{2} d}\right]$ and $(2 p, n)=1$.

The implication $(2) \Rightarrow(3)$ is obvious. Hence it suffices to prove $\left(1^{\prime}\right)$ $\Rightarrow(2)$ and $(3) \Rightarrow\left(1^{\prime}\right)$.

$\left(1^{\prime}\right) \Rightarrow(2)$ : It follows directly from [4], (3.6) that $C(Z \Pi) / C^{q}(Z \Pi)=$ $C\left(\Omega_{Z I}\right)$. Therefore, by (1.5) and (3.1), it suffices to show that $D_{\Pi}=L_{\Pi}$. In case $\left(\mathrm{i}^{\prime}\right) \ell=\ell(\Pi)=|\Pi|$ and $m=m(\Pi)=1$, while, in case (ii'), $\ell=$ $\ell(\Pi)=n p^{c}, m=m(\Pi)=2^{d}$ and $i(\Pi)=2$. Let $\sigma$ be an element of $\Pi$ of order $\ell$. In case ( $\mathrm{i}^{\prime}$ ) we define $\mu=\sigma$ and $b=\ell$. On the other hand, in case (ii'), we define $\mu=\sigma \tau^{2}$ and $b=\ell 2^{d-1}$. Then $\langle\mu\rangle$ is the normal subgroup of $\Pi$ of order $b$. Now, by induction on $b$, we will prove that $D_{\Pi}=L_{\Pi}$. For every $b^{\prime} \mid b$, let $\Psi_{b^{\prime}}(X)=X^{b}-1 / X^{b^{\prime}}-1$. Then we can construct the following exact sequences:

$$
\begin{aligned}
& 0 \longrightarrow Z \Pi /\left(\Psi^{(1)}(\mu)\right) \longrightarrow Z \Pi /\left(\chi^{(0)}(\mu)\right) \longrightarrow Z \Pi /\left(\mu^{b_{1}}-1\right) \longrightarrow 0 \text {, } \\
& 0 \longrightarrow Z \Pi /\left(\Psi^{(1)}(\mu)\right) \longrightarrow Z \Pi /\left(\chi^{(1)}(\mu)\right) \longrightarrow Z \Pi /\left(\mu^{b_{2}}-1\right) \longrightarrow 0 \text {, } \\
& 0 \longrightarrow Z \Pi /\left(\Psi^{(2)}(\mu)\right) \longrightarrow Z \Pi /\left(\chi^{(1)}(\mu)\right) \longrightarrow Z \Pi /\left(\mu^{b_{3}}-1\right) \longrightarrow 0 \text {, } \\
& \text {............. } \\
& 0 \longrightarrow Z \Pi /\left(\Psi^{(t-1)}(\mu)\right) \longrightarrow Z \Pi /\left(\chi^{(t-1)}(\mu)\right) \longrightarrow Z \Pi /\left(\mu^{b_{2 t-2}}-1\right) \longrightarrow 0 \text {, } \\
& 0 \longrightarrow Z \Pi /\left(\Psi^{(t)}(\mu)\right) \longrightarrow Z \Pi /\left(\chi^{(t-1)}(\mu)\right) \longrightarrow Z \Pi /\left(\mu^{b_{2 t-1}}-1\right) \longrightarrow 0 \text {, }
\end{aligned}
$$

where, for $1 \leqq i \leqq 2 t-1, b_{i} \| b$ and, for $1 \leqq j \leqq t-1, \Psi^{(j)}(X) \mid \Psi_{b_{2 j-1}}(X)$, $\Psi^{(j)}(X) \mid \Psi_{b_{2 j}}(X), \quad \chi^{(j)}(X)=\Psi^{(j)}(X)\left(X^{b_{2 j}}-1\right)=\Psi^{(j+1)}(X)\left(X^{b_{2 j+1}}-1\right), \chi^{(0)}(X)=$ $X^{b}-1$ and $\Psi^{(t)}(X)=\Phi_{b}(X)$. Let $M \in D_{m}$. Then, for any $b^{\prime} \mid b, M /\left(\mu^{b^{\prime}}-\right.$ 1) $M \in D_{\Pi /\left\langle\mu^{b^{\prime}}\right\rangle}$. For any $\Psi(X) \mid X^{b}-1$ we define $\bar{M}_{\Psi}=M / \Psi(\mu) M / t(M / \Psi(\mu) M)$. Tensoring the above exact sequences with $M$ over $Z \Pi$, we get the exact sequences :

$$
\begin{aligned}
& 0 \longrightarrow \bar{M}_{\Psi(1)} \longrightarrow M \longrightarrow M /\left(\mu^{b_{1}}-1\right) M \longrightarrow 0 \text {, } \\
& 0 \longrightarrow \bar{M}_{\Psi(1)} \longrightarrow \bar{M}_{\chi^{(1)}} \longrightarrow M /\left(\mu^{b_{2}}-1\right) M \longrightarrow 0 \text { ， } \\
& 0 \longrightarrow \bar{M}_{\Psi(2)} \longrightarrow \bar{M}_{\chi^{(1)}} \longrightarrow M /\left(\mu^{b_{3}}-1\right) M \longrightarrow 0 \text {, } \\
& 0 \longrightarrow \bar{M}_{\Psi(t-1)} \longrightarrow \bar{M}_{\chi^{(t-1)}} \longrightarrow M /\left(\mu^{b_{2 t-2}}-1\right) M \longrightarrow 0,
\end{aligned}
$$




$$
0 \longrightarrow \bar{M}_{\Phi_{b}} \longrightarrow \bar{M}_{\chi^{(t-1)}} \longrightarrow M /\left(\mu^{b_{2 t-1}}-1\right) M \longrightarrow 0 .
$$

Since $M /\left(\mu^{b_{i}}-1\right) M \in D_{\Pi /\left\langle\mu^{b i\rangle}\right.}$, applying (1.3) repeatedly, we get

$$
M \oplus \bigoplus_{j=1}^{t-1}\left(M /\left(\mu^{b_{2 j}}-1\right) M\right) \overline{\overline{(z)}} \bar{M}_{\Phi_{b}} \oplus \bigoplus_{j=1}^{t}\left(M /\left(\mu^{b_{2 j-1}}-1\right) M\right) .
$$

By induction on $b$ we have $M /\left(\mu^{b_{i}}-1\right) M \in L_{I /\left\langle\mu^{\left.b_{i}\right\rangle}\right.} \subseteq L_{I I}$ for each $1 \leqq i \leqq$ $2 t-1$. Accordingly we only need to show that $\bar{M}_{\Phi_{b}} \overline{(z)} \mathfrak{A}$ for some $\mathfrak{U} \in I_{\Pi}$. In case $\left(\mathrm{i}^{\prime}\right) Z \Pi /\left(\Phi_{b}(\mu)\right) \cong Z\left[\zeta_{b}\right]$, and therefore there exist $\mathfrak{U} \in \boldsymbol{I}_{I}$ and $k \geqq 0$ such that $\bar{M}_{\Phi_{b}} \oplus Z \Pi \cong Z\left[\zeta_{b}\right]^{(k)} \oplus \mathfrak{A}$. Hence $\bar{M}_{\Phi_{b}} \frac{}{(\mathfrak{*})} \mathfrak{A}$. In case (ii') we put $\Lambda=Z \Pi /\left(\Phi_{b}(\mu)\right)$. Then $\Lambda \frac{(r)}{-} 0$. If $d=1, \Lambda$ is isomorphic to the trivial crossed product of $Z\left[\zeta_{b}\right]$ and $\langle\tau\rangle$. Let $R=Z\left[\zeta_{b}\right]$ and $\mathfrak{P}=$ $\left(\zeta_{p c}-1\right)$. Then both $R$ and $\mathfrak{P}$ can regarded as $\Lambda$-modules. By assumption $\mathfrak{P}$ is the unique prime ideal of $R$ ramified over $Z\left[\zeta_{n}\right] \subseteq Z\left[\zeta_{b}\right]^{\langle\tau\rangle}$. Therefore $\Lambda \cong R \oplus \mathfrak{P}$ as $\Lambda$-modules and any ambiguous ideal of $R$ has the same genus as that of $R$ or $\mathfrak{\beta}$. Hence there exist $\mathfrak{Y} \in I_{I}$ and $u, v \geqq 0$ such that $\bar{M}_{\Phi_{b}} \oplus Z \Pi \cong R^{(u)} \oplus \mathfrak{P}^{(v)} \oplus \mathfrak{A}$. Since $R-\frac{}{{ }^{(r)}} 0$ and $\mathfrak{P} \frac{{ }_{(x)}}{-} 0$, this

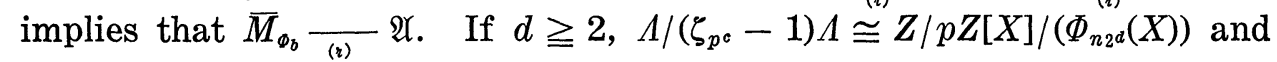
so $\Lambda /\left(\zeta_{p c}-1\right) \Lambda$ is a field because $p$ is a prime in $Z\left[\zeta_{n 2^{d}}\right]$. Therefore $\Lambda$ is a maximal order in $Q \Lambda$ and $Q \Lambda$ is a division ring. Then we have $\bar{M}_{\Phi_{b}} \oplus Z \Pi \cong \Lambda^{(u)} \oplus \mathfrak{A}$ for some $\mathfrak{U} \in I_{I}$ and $u \geqq 0$ and so $\bar{M}_{\Phi_{b}}-\mathfrak{A}$. Thus the proof of $\left(1^{\prime}\right) \Rightarrow(2)$ is completed.

$(3) \Rightarrow\left(1^{\prime}\right)$ : Assume that $I I$ does not satisfy the condition $\left(1^{\prime}\right)$. We will prove that $T(\Pi)$ is not a finite group. If $\Pi$ has a noncyclic Sylow subgroup, this follows from (1.5). Hence we may assume that every Sylow subgroup of $\Pi$ is cyclic. If $\mathrm{i}(\Pi)>2$, it follows from (2.3) that $T(I)$ is not finite. Therefore we may further assume that $i(I) \leqq 2$. If $T(\Pi)$ is a finite group, then, for any normal subgroup $\Pi^{\prime}$ of $\Pi, T\left(\Pi / \Pi^{\prime}\right)$ is a finite group. Hence we only need to show that $T(I)$ is not finite, in each of the following cases:

(a) $I I$ is a group with generators $\sigma, \tau$ and relations $\sigma^{p_{1} p_{2}}=\tau^{2}=I$ and $\tau^{-1} \sigma \tau=\sigma^{-1}$ where $p_{1}$ and $p_{2}$ are distinct odd primes.

(b) $\Pi$ is a group with generators $\sigma$, $\tau$ and relations $\sigma^{n p}=\tau^{2^{d}}=I$, $d \geqq 1, \tau^{-1} \sigma^{n} \tau=\sigma^{-n}$ and $\sigma^{p} \tau=\tau \sigma^{p}$ where $p$ is an odd prime which is not a prime in $Z\left[\zeta_{n^{d}}\right]$ and $(2 p, n)=1$.

We define $\mu=\sigma$ and $b=p_{1} p_{2}$ in case (a) and $\mu=\sigma \tau^{2}$ and $b=n p 2^{d-1}$ in case (b). Let $\Lambda=Z \Pi /\left(\Phi_{b}(\mu)\right)$. If $\Pi$ has the type (a) or the type (b), $d=1$, then $\Lambda$ is isomorphic to the trivial crossed product of $Z\left[\zeta_{b}\right]$ and $\langle\tau\rangle$. 
Further let $R=Z\left[\zeta_{b}\right]$. Then $A \frac{(z)}{(z)}$ and $R-\frac{}{(z)} 0$. There is an ambiguous ideal $\cong$ of $R$ such that $\Lambda \cong R \oplus \Omega$. Then we also have $\cong \frac{(n)}{(n)}$. If $T \in S_{I I}, \Lambda T \cong R^{(u)} \oplus \mathfrak{Q}^{(v)}$ for some $u, v \geqq 0$. In each case we can find an ambiguous ideal $\subseteq$ of $R$ whose genus is different from those of $R$

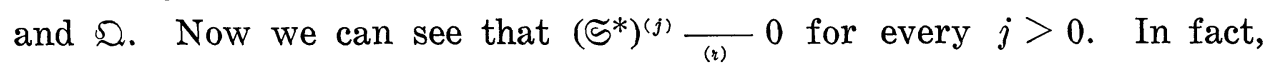
if $\left(\mathfrak{S}^{*}\right)^{(j)} \frac{-}{(z)} 0$, there is an exact sequence $0 \rightarrow S^{\prime} \rightarrow S \rightarrow \mathbb{S}^{(j)} \rightarrow 0$ of $\Pi$-modules with $S^{\prime}, S \in \boldsymbol{S}_{\Pi}$. Tensoring this with $\Lambda$ over $Z \Pi$ and eliminating the torsion parts, we get $\mathbb{S}^{(j)} \oplus \Lambda S^{\prime} \cong \Lambda S$ and so $\mathbb{S}^{(j)} \oplus R^{(u)} \oplus \mathfrak{Q}^{(v)} \cong R^{\left(u^{\prime}\right)}$ $\oplus \Omega^{\left(v^{\prime}\right)}$ for some $u, v, u^{\prime}, v^{\prime} \geqq 0$, which is a contradiction. Thus $T(I)$ is not a finite group. Finally suppose that $\Pi$ has the type (b) and $d \geqq 2$. Since $p$ is not a prime in $Z\left[\zeta_{n 2^{d}}\right], \Lambda$ is a non-maximal hereditary order in Q1. There is an ideal $\mathfrak{Q}$ of $\Lambda$ whose genus is different from that of $\Lambda$ such that $Q \cong \cong Q \Lambda$. It is easily seen that, if $\Pi^{\prime}$ is a proper subgroup of $\Pi$, then $\Lambda \cdot Z \Pi / \Pi^{\prime}=0$. Hence we can show that $\left(\Omega^{*}\right)^{(j)} \frac{(k)}{\left(_{(2)}\right.} 0$ for every $j>0$, and so $T(\Pi)$ is not a finite group. This completes the proof of $(3) \Rightarrow\left(1^{\prime}\right)$.

§4. Let $\Gamma$ be a ring with unit element. We denote by $G(\Gamma)$ the Grothendieck group of the category of finitely generated $\Gamma$-modules. Let $I I$ be a finite group. Then there is a natural epimorphism $\nu_{\Pi}: G(Z I)$ $\rightarrow G(Q \Pi)$. There is also a natural homomorphism $\omega_{I I}: C\left(\Omega_{Z I}\right) \rightarrow G(Z \Pi)$. It was shown in [11] that the sequence $C\left(\Omega_{Z I}\right) \stackrel{\omega_{I}}{\longrightarrow} G(Z \Pi) \stackrel{\nu_{I I}}{\longrightarrow} G(Q \Pi)$ $\rightarrow 0$ is exact.

Let $K$ be an algebraic number field and let $R$ be the ring of all algebraic integers in $K$. Let $\Sigma$ be a central simple $K$-algebra. We denote the completion of $K$ at a valuation $v$ of $K$ by $\hat{K}_{v}$. It is said that an archimedian valuation $v$ of $K$ is ramified in $\Sigma$ if $\hat{K}_{v} \otimes_{K} \Sigma$ is isomorphic to a full matrix algebra over the quaternion field. Denote by $I_{K}$ the group of all fractional ideals in $K$ and define $P_{\Sigma}^{\prime}=\left\{\alpha R \in I_{K} \mid v(\alpha)>0\right.$ at every archimedian valuation $v$ ramified in $\Sigma$ \}. Let $\Omega$ be a maximal $R$ order in $\Sigma$. It was proved in $[10]$ that $C(\Omega) \cong I_{K} / P_{\Sigma}^{\prime}$.

Suppose that $\Pi$ is a finite group whose Sylow subgroups are cyclic. Let $\Sigma$ be a simple component of QII. Let $K_{\Sigma}$ be the center of $\Sigma$ and let $R_{\Sigma}$ be the ring of all algebraic integers in $K_{\Sigma}$. Define $\Pi_{\Sigma}=\operatorname{Ker}(I I$ $\rightarrow \Sigma$ ) and let $\tilde{\Pi}_{\Sigma}=\left\langle\sigma_{\Sigma}\right\rangle$ be the maximal normal cyclic subgroup of $\Pi / \Pi_{\Sigma}$. Further put $a_{\Sigma}=\left|\Pi_{\Sigma}\right|\left|\tilde{\Pi}_{\Sigma}\right|$ and $b_{\Sigma}=\left|\tilde{\Pi}_{\Sigma}\right|$. We have $\Sigma \cong Q \Pi / \Pi_{\Sigma} /\left(\Phi_{b_{\Sigma}}\left(\sigma_{\Sigma}\right)\right)$ and so $\Sigma$ can be expressed as a crossed product of $Q\left(\zeta_{b_{\Sigma}}\right)$ and $\Pi / \Pi_{\Sigma} /\left\langle\sigma_{\Sigma}\right\rangle$.

LeMma 4.1. Let $\Pi$ be a finite group whose Sylow subgroups are 
cyclic.

(1) If, for each simple component $\Sigma$ of $Q \Pi$, every prime divisor of $a_{\Sigma}$ in $R_{\Sigma}$ is contained in $P_{\Sigma}^{\prime}$, then $\omega_{\Pi}: C\left(\Omega_{Z_{\Pi}}\right) \rightarrow G(Z \Pi)$ is a monomorphism.

(2) If $\omega_{I I}: C\left(\Omega_{Z I}\right) \rightarrow G(Z I)$ is a monomorphism, then, for each simple component $\Sigma$ of $\Omega \Pi$, every prime divisor of $b_{\Sigma}$ in $R_{\Sigma}$ is contained in $P_{\Sigma}^{\prime}$.

Proof. (1) It suffices to prove that, for any exact sequence $0 \rightarrow M^{\prime}$ $\rightarrow M \rightarrow M^{\prime \prime} \rightarrow 0$ of $\Pi$-modules, $\Omega_{Z I} M \oplus \Omega_{Z I} \cong \Omega_{Z I I} M^{\prime} \oplus \Omega_{Z I} M^{\prime \prime} \oplus \Omega_{Z I}$. Let $\Sigma$ be any simple component of $Q \Pi$, and let $\Lambda$ and $\Omega$ be the images of $Z \Pi$ and $\Omega_{z \Pi}$ under the projection $Q \Pi \rightarrow \Sigma$, respectively. Now we will show that $\Omega M \oplus \Omega \cong \Omega M^{\prime} \oplus \Omega M^{\prime \prime} \oplus \Omega$. Taking the cohomology as $\Pi_{\Sigma^{-}}$ modules, we have the exact sequence:

$$
0 \longrightarrow M^{\prime I_{\Sigma}} \longrightarrow M^{n_{\Sigma}} \stackrel{f}{\longrightarrow} M^{\prime \Pi_{\Sigma}} \stackrel{g}{\longrightarrow} H^{1}\left(I I_{\Sigma}, M^{\prime}\right) .
$$

This yields the exact sequences.

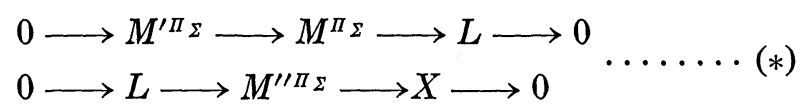

where $L=\operatorname{Im} f, X=\operatorname{Im} g$ and $\left|\Pi_{\Sigma}\right| X=0$. Let $0 \rightarrow N^{\prime} \rightarrow N \rightarrow Y \rightarrow 0$ be an exact sequence where $N^{\prime}, N$ are $\Pi / \Pi_{\Sigma}$-modules and $a_{\Sigma}^{s} Y=0$ for some $s>0$. Then $a_{\Sigma}^{s} \Omega N \subseteq \Omega N^{\prime}$. Since $C(\Omega) \cong I_{k} / P_{\Sigma}^{\prime}$ and every prime divisor of $a_{\Sigma}$ in $K$ is contained in $P_{\Sigma}^{\prime}$, we have $\Omega N \oplus \Omega \cong \Omega N^{\prime} \oplus \Omega$. Let $\Psi_{\Sigma}=\sum_{\tau \in \Pi_{\Sigma}} \tau$. For any $\Pi$-module $N \Psi_{\Sigma} N \subseteq N^{\Pi_{\Sigma}}$ and $\left|\Pi_{\Sigma}\right| N^{\Pi_{\Sigma}} \subseteq \Psi_{\Sigma} N$, and so $\Omega N^{\Pi_{\Sigma}} \oplus \Omega \cong \Omega \Psi_{\Sigma} N \oplus \Omega$. As is easily seen, $\Omega \Psi_{\Sigma} N \cong \Omega N$. Thus $\Omega N \oplus \Omega \cong \Omega N^{I_{\Sigma}} \oplus \Omega$. Applying these to (*), we get $\Omega M^{\prime} \oplus \Omega \cong \Omega M^{\prime \Pi_{\Sigma}} \oplus \Omega$, $\Omega M \oplus \Omega \cong \Omega M^{\Pi_{\Sigma}} \oplus \Omega$ and $\Omega M^{\prime \prime} \oplus \Omega \cong \Omega M^{\prime I_{\Sigma}} \oplus \Omega \cong \Omega L \oplus \Omega$. Hence we only need to prove that $\Omega M^{\Pi_{\Sigma}} \oplus \Omega \cong \Omega M^{\prime \Pi_{\Sigma}} \oplus \Omega L \oplus \Omega$. Therefore it suffices to prove, under the assumption that $\Pi_{\Sigma}=\{I\}$, that $\Omega M \oplus \Omega \cong \Omega M^{\prime} \oplus \Omega M^{\prime \prime} \oplus \Omega$. To simplify our notation we write $\sigma=\sigma_{\Sigma}, b=b_{\Sigma}, K=K_{\Sigma}$ and $R=R_{\Sigma}$. If $\Pi$ is cyclic, then $\Lambda=\Omega$. On the other hand, if $\Pi$ is not cyclic, then $\Lambda$ is a crossed product of $Z\left[\zeta_{b}\right]$ and $\Pi /\langle\sigma\rangle$ and therefore, for every prime ideal $\mathfrak{p}$ of $R$ which does not contain $b, \Lambda_{\mathfrak{p}}=\Omega_{\mathfrak{p}}$. Since $|\Pi| \Omega_{Z \Pi} \subseteq Z \Pi$, we can find $t>0$ such that $b^{t} \Omega \subseteq \Lambda$. Let $N$ be a $\Pi$-module and define $N^{\Phi_{b}}=\left\{u \in N \mid \Phi_{b}(\sigma) u=0\right\}$. Then $N^{\Phi_{b}} \subseteq \Lambda N$ and $b \Lambda N \subseteq N^{\Phi_{b}}$, and therefore $\Omega N \oplus \Omega \cong \Omega N^{\Phi_{b}} \oplus \Omega$. Returning to the sequence $0 \rightarrow M^{\prime} \rightarrow M \rightarrow M^{\prime \prime} \rightarrow 0$, we get the exact sequence:

$$
0 \longrightarrow M^{\prime \Phi_{b}} \longrightarrow M^{\Phi_{b}} \longrightarrow M^{\prime \prime \Phi_{b}} \longrightarrow U \longrightarrow 0
$$


of $\Lambda$-modules where $b U=0$. This can be separated into two short exact sequences :

$$
\begin{aligned}
& 0 \longrightarrow M^{\prime \Phi_{b}} \longrightarrow M^{\Phi_{b}} \longrightarrow L \longrightarrow 0, \\
& 0 \longrightarrow L \longrightarrow M^{\prime \Phi_{b}} \longrightarrow U \longrightarrow 0
\end{aligned}
$$

Since $b^{t} \Omega \subseteq \Lambda$ and $b U=0, \Omega M^{\Phi_{b}} \oplus \Omega \cong \Omega M^{\prime \Phi_{b}} \oplus \Omega L \oplus \Omega \cong \Omega M^{\prime \Phi_{b}} \oplus \Omega M^{\prime \prime \Phi_{b}}$ $\oplus \Omega$. However $\Omega M \oplus \Omega \cong \Omega M^{\Phi_{b}} \oplus \Omega, \Omega M^{\prime} \oplus \Omega \cong \Omega M^{\prime \Phi_{b}} \oplus \Omega$ and $\Omega M^{\prime \prime} \oplus \Omega$ $\cong \Omega M^{\prime \prime \Phi_{b}} \oplus \Omega$. Thus $\Omega M \oplus \Omega \cong \Omega M^{\prime} \oplus \Omega M^{\prime \prime} \oplus \Omega$.

(2) Let $\Sigma$ be a simple component of $Q \Pi$ and let $\mathfrak{p}$ be a prime divisor of $b_{\Sigma}$ in $R_{\Sigma}$. In order to show that $\mathfrak{p} \in P_{\Sigma}^{\prime}$, we may assume that $\Pi_{\Sigma}=$ $\{I\}$, and hence we can use the notation as in the proof of (1). There is an exact sequence

$$
0 \longrightarrow N \longrightarrow \Omega \longrightarrow X \longrightarrow 0
$$

of $\Omega$-modules where $X$ is a simple $\Omega$-module such that $\mathfrak{p} X=0$. Since $C(\Omega)=I_{K} / P_{\Sigma}^{\prime}$, we only need to prove that $N \oplus \Omega \cong \Omega \oplus \Omega$. Let $p$ be a rational prime such that $p Z=\mathfrak{p} \cap Z$ and let $b=p^{c} b^{\prime}, p \times b^{\prime}$. Further let $\Sigma^{\prime}=Q \Pi /\left(\Phi_{b},(\sigma)\right)$ and let $\Lambda^{\prime}$ and $\Omega^{\prime}$ be the images of $Z \Pi$ and $\Omega_{z \Pi}$ under the projection $Q \Pi \rightarrow \Sigma^{\prime}$, respectively. Then $\Lambda^{\prime}=Z \Pi /\left(\Phi_{b}(\sigma)\right)$. Since $\Phi_{b}\left(\zeta_{b}\right) \in \mathfrak{p}, X$ can be regarded as a $\Lambda^{\prime}$-module. We see that $\Lambda_{p}^{\prime}=\Omega_{p}^{\prime}$ and so $X$ can be regarded as a $\Omega^{\prime}$-module. Therefore there is an exact sequence

$$
0 \longrightarrow N^{\prime} \longrightarrow \Omega^{\prime(t)} \longrightarrow X \longrightarrow 0, t>0
$$

of $\Omega^{\prime}$-modules. Forming the pullback $\Omega \rightarrow X$ we get $\Omega \oplus N^{\prime} \cong N \oplus \Omega^{\prime(t)}$.<smiles>C1CC[GeH2]C1</smiles>

Since $\omega_{\Pi}: C\left(\Omega_{Z I}\right) \rightarrow G(Z \Pi)$ is a monomorphism, the natural epimorphism $G\left(\Omega_{Z I}\right) \rightarrow G(Z \Pi)$ is an isomorphism ([11], (5.4)). Then $G(\Omega) \oplus G\left(\Omega^{\prime}\right)$ is the direct summand of $G\left(\Omega_{z I}\right)$ and so $G(\Omega) \cap G\left(\Omega^{\prime}\right)=0$ in $G\left(\Omega_{z I}\right)$. Hence the fact that $\Omega \oplus N^{\prime} \cong N \oplus \Omega^{\prime(t)}$ implies that $N \oplus \Omega \cong \Omega \oplus \Omega$ and $N^{\prime} \oplus$ $\Omega^{\prime} \cong \Omega^{\prime(t+1)}$. Thus the proof of (2) is completed.

We denote by $B_{G}(Z \Pi)$ the subgroup of $G(Z \Pi)$ generated by all the images of permutation $\Pi$-modules in $G(Z \Pi)$ and define $S w(\Pi)=$ $G(Z \Pi) / B_{G}(Z \Pi)$. Then there exists a natural homomorphism $\theta_{\Pi}: \dot{T}^{g}(I)$ $\rightarrow S w(\Pi)$. We will denote the image of a $\Pi$-module $L$ in $G(Z \Pi)$ (resp. $S w(\Pi))$ by $\langle L\rangle$ (resp. $\langle L L\rangle)$. 
THEOREM 4.2. The following statements on a finite group $I$ are equivalent:

(1). $\Pi$ is one of the following groups: (i) a cyclic group of order $n$ where for every $m \mid n$ any prime ideal of $Z\left[\zeta_{m}\right]$ containing $n$ is principal. (ii) a dihedral group of order $2 p^{c}$ where $c \geqq 1$ and $p$ is an odd prime. (iii) a direct product of a cyclic group of order $q^{f}$ and a dihedral group of order $2 p^{c}$ where $f, c \geqq 1, p$ and $q$ are odd primes, $p$ is a primitive $q^{f-1}(q-1)$-th root of unity modulo $q^{f}$, for every $1 \leqq f^{\prime} \leqq f$ any prime ideal of $Z\left[\zeta_{q f^{\prime}}\right]$ containing 2 is principal and for every $1 \leqq f^{\prime} \leqq f$ and every $1 \leqq c^{\prime} \leqq c$ any prime ideal of $Z\left[\zeta_{q f^{\prime}}, \zeta_{p^{\prime}}+\zeta_{p^{\prime}}^{-1}\right]$ containing $q$ is principal. (iv) a generalized quaternion group of order $4 p^{c}$ where $c \geqq 1$, $p$ is an odd prime congruent to 3 modulo 4 and for every $1 \leqq c^{\prime} \leqq c$ any prime ideal of $Z\left[\zeta_{p c^{\prime}}+\zeta_{p^{\prime}}^{-1}\right]$ containing 2 is generated by a totally positive element.

(2) $T(\Pi)=C\left(\Omega_{Z_{\Pi}}\right)$ and $\omega_{\Pi}: C\left(\Omega_{Z \Pi}\right) \rightarrow G(Z \Pi)$ is a monomorphism.

(3) $T(\Pi)=T^{g}(\Pi)$ and $\theta_{\Pi}: T(\Pi) \rightarrow S w(\Pi)$ is an isomorphism.

(4) The dual module of a quasi-permutation $\Pi$-module is always a quasi-permutation $\Pi$-module.

(5) If $0 \rightarrow M^{\prime} \rightarrow M \rightarrow M^{\prime \prime} \rightarrow 0$ is an exact sequence of $\Pi$-modules and any two of $M^{\prime}, M, M^{\prime \prime}$ are quasi-permutation $\Pi$-modules, then the third one is a quasi-permutation $\Pi$-module.

Proof. From (3.3) and (4.1) it follows immediately that (1) and (2) are equivalent. The implications $(3) \Rightarrow(5) \Rightarrow(4)$ are obvious. Hence we only need to prove $(1) \Rightarrow(3),(3) \Rightarrow(2)$ and $(4) \Rightarrow(3)$.

$(1) \Rightarrow(3)$ : We suppose that $I$ satisfies (1). Then by (1.5) and (3.3)

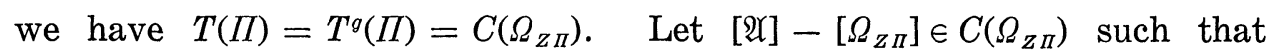
$\theta_{I I}\left([\mathfrak{V}]-\left[\Omega_{Z I}\right]\right)=0$, i.e., $\langle\langle\mathfrak{U}\rangle\rangle=\left\langle\left\langle\Omega_{Z_{I}}\right\rangle\right\rangle$. We will show that $\mathfrak{U} \oplus \Omega_{Z_{I}} \cong$ $\Omega_{z \Pi} \oplus \Omega_{z I}$. There exist $S_{1}, S_{2} \in S_{I I}$ such that $\left\langle\mathfrak{U} \oplus S_{1}\right\rangle=\left\langle\Omega_{Z I} \oplus S_{2}\right\rangle$. Hence we have exact sequences

$$
\begin{aligned}
& 0 \longrightarrow L^{\prime} \longrightarrow \mathfrak{A} \oplus S_{1} \oplus L \longrightarrow L^{\prime \prime} \longrightarrow 0, \\
& 0 \longrightarrow L^{\prime} \longrightarrow \Omega_{Z I I} \oplus S_{2} \oplus L \longrightarrow L^{\prime \prime} \longrightarrow 0
\end{aligned}
$$

with $L^{\prime}, L, L^{\prime \prime} \in M_{\Pi}$. Therefore, by virtue of (4.1), we have

$$
\mathfrak{U} \oplus \Omega_{Z \Pi} S_{1} \cong \Omega_{Z I I} \oplus \Omega_{z \Pi} S_{2} .
$$

Let $\Sigma$ be a simple component of $Q \Pi$ and let $\Lambda$ and $\Omega$ be the images of $Z \Pi$ and $\Omega_{Z \Pi}$ under the projection $Q \Pi \rightarrow \Sigma$, respectively. Now it suffices 
to show that $\Omega \mathfrak{A} \oplus \Omega \cong \Omega \oplus \Omega$. If $\Sigma$ is a division ring, then $\Omega S_{1} \cong \Omega S_{2}$ $\cong \Omega^{(t)}$ for some $t \geqq 0$ so that $\Omega \mathscr{U} \oplus \Omega \cong \Omega \oplus \Omega$. On the other hand, if $\Sigma$ is not a division ring, then $\Lambda$ is the trivial crossed product of $Z$ [ $\left.\zeta_{q f^{\prime} p c^{\prime}}\right]$ and a cyclic group $\langle\tau\rangle$ of order 2 where $0 \leqq f^{\prime} \leqq f$ and $1 \leqq c^{\prime}$, because $\Pi$ is one of the groups as in (1). Let $R=Z\left[\zeta_{q f^{\prime} p^{\prime}}\right]$ and $\Re=\left(\zeta_{p^{\prime}}-1\right)$. Then $\mathfrak{\beta}$ is a prime ideal of $R$. Both $R$ and $\mathfrak{\beta}$ can be regarded as $\Lambda$ modules and $\Lambda \cong R \oplus \Re$ as $\Lambda$-modules. Further we see that $\Lambda S_{1} \cong \Lambda^{\left(u_{1}\right)}$ $\oplus R^{\left(v_{1}\right)}$ and $\Lambda S_{2} \cong \Lambda^{\left(u_{2}\right)} \oplus R^{\left(v_{2}\right)}$ for some $u_{1}, v_{1}, u_{2}, v_{2} \geqq 0$. Consider the exact sequence $0 \rightarrow \mathfrak{B} \rightarrow R \rightarrow R / \mathfrak{R} \rightarrow 0$. Since $p \cdot(R / \mathfrak{P})=0$, we get $\Omega R \cong \Omega \mathfrak{P}$ and so $\Omega \cong \Omega R \oplus \Omega \Re \cong \Omega R \oplus \Omega R$. Hence we have $\Omega S_{1} \cong \Omega R^{\left(u_{1}+v_{1}\right)}$ and $\Omega S_{2} \cong \Omega R^{\left(u_{2}+v_{2}\right)}$. Therefore $\Omega \mathscr{U} \oplus \Omega R^{\left(u_{1}+v_{1}\right)} \cong \Omega \oplus \Omega R^{\left(u_{2}+v_{2}\right)}$. Thus we can conclude that $\Omega \mathscr{U} \cong \Omega$.

$(3) \Rightarrow(2)$ : Suppose that $T(\Pi)=T^{g}(\Pi)$ and $\theta_{\Pi}: T(\Pi) \rightarrow S w(\Pi)$ is an isomorphism. Since $S w(\Pi)$ is a finite group, $T(\Pi)$ is so. Then by (3.3) we have $T(\Pi)=C\left(\Omega_{Z I}\right)$. Considering the commutative diagram

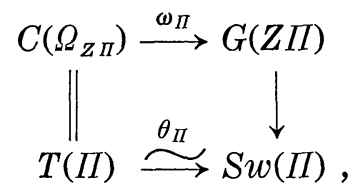

we see that $\omega_{I I}: C\left(\Omega_{z \Pi}\right) \rightarrow G(Z \Pi)$ is a monomorphism.

(4) $\Rightarrow(3)$ : Suppose that $\Pi$ satisfies (4). Then we have by (1.5) $T(\Pi)$ $=T^{g}(\Pi)$. Let $[M] \in T(\Pi)$ such that $\theta_{\Pi}([M])=\langle\langle M\rangle=0$. Then there exist $S^{\prime}, S \in S_{\Pi}$ such that $\left\langle M \oplus S^{\prime}\right\rangle=\langle S\rangle$. Hence we have exact sequences

$$
\begin{aligned}
& 0 \longrightarrow L^{\prime} \longrightarrow M \oplus S^{\prime} \oplus L \longrightarrow L^{\prime \prime} \longrightarrow 0, \\
& 0 \longrightarrow L^{\prime} \longrightarrow S \oplus L \longrightarrow L^{\prime \prime} \longrightarrow 0
\end{aligned}
$$

with $L^{\prime}, L, L^{\prime \prime} \in M_{I I}$. Here we may assume by (1.5) that $L^{\prime} \frac{-}{{ }^{(z)}} 0$ and

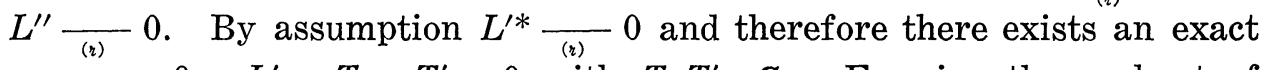
sequence $0 \rightarrow L^{\prime} \rightarrow T \rightarrow T^{\prime} \rightarrow 0$ with $T, T^{\prime} \in S_{\Pi}$. Forming the pushout of $L^{\prime} \rightarrow S \oplus L$, we get the following commutative diagram with exact rows $\stackrel{\downarrow}{T}$

and columns : 


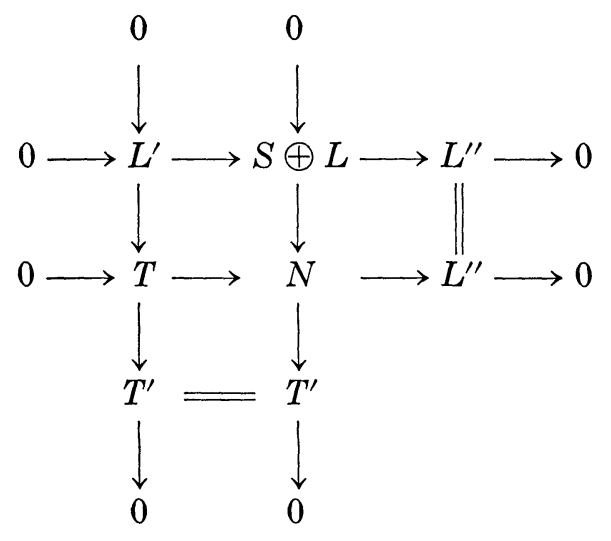

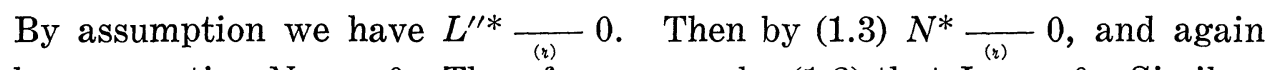
by assumption $N \frac{}{(z)} 0$. Therefore we see by (1.3) that $L \frac{(z)}{\left(^{(z)}\right.} 0$. Similarly, forming the pushout of $L^{\prime} \rightarrow M \oplus S^{\prime} \oplus L$, we can show that $M \oplus L$ $\downarrow$

$\overline{\theta^{(z)}} 0$. Consequently we have $M \frac{}{(z)} M \oplus L \frac{}{(z)} 0$. This proves that $\theta_{\Pi}: T(I) \rightarrow S w(I)$ is an isomorphism.

Let $\Pi=\langle\sigma\rangle$ be a cyclic group of order $n$ and let $L$ be a $\Pi$-module. For every $m \mid n$ we define $L^{\Phi_{m}}=\left\{u \in L \mid \Phi_{m}(\sigma) u=0\right\}$.

COROLLARY 4.3. Let II be a cyclic group of order $n$. If II satisfies the conditions in (4.2), then it satisfies the following condition:

(6) A II-module $M$ is a quasi-permutation II-module if and only if, for every $m \mid n, M^{\Phi_{m}}$ is a free $Z\left[\zeta_{m}\right]$-module.

Proof. It has been shown in the proof of (4.2) that if $L$ is a $\Pi$ module then for every $m \mid n L^{\Phi_{m}} \cong Z\left[\zeta_{m}\right] L$. Hence the only if part follows immediately from (4.1). Let $M$ be a $\Pi$-module such that for every $m \mid n$ $M^{\Phi_{m}}$ is $Z\left[\zeta_{m}\right]$-free. By (1.5) and (3.3) there is a projective ideal $\mathfrak{A}$ of $Z \Pi$ such that $M \oplus \mathfrak{A} \frac{}{{ }^{(z)}} 0$. Then $M^{\Phi_{m}} \oplus \mathfrak{Q}^{\Phi_{m}}$ is $Z\left[\zeta_{m}\right]$-free, hence $\mathfrak{Q}^{\Phi_{m}}$ is so. Therefore by [3], (2.5) $\mathfrak{U} \frac{(z)}{(z)}$. Thus $M \frac{(z)}{-} 0$.

(4.2) and (4.3) show that the conjecture given in [3], p. 416 is true. It should be noted that the converse to (4.3) is not true ([3]).

\section{REFERENCES}

[1] C. Chevalley, On algebraic group varieties, J. Math. Soc. Japan, 6 (1954), 303-324. [2] S. Endo and T. Miyata, Invariants of finite abelian groups, J. Math. Soc. Japan, 25 (1973), 7-26. 
[3] — Quasi-permutation modules over finite groups, J. Math. Soc. Japan, 25 (1973), 397-421.

[ 4 ] — Q Quasi-permutation modules over finite groups, II, J. Math. Soc. Japan, 26 (1974), 698-713.

[5] H. Jacobinski, Genera and decompositions of lattices over orders, Acta Math., 121 (1968), 1-29.

[6] H. W. Lenstra Jr., Rational functions invariant under a finite abelian group, Preprint, Univ. Amsterdam, 1972.

[ 7 ] T. Ono, Arithmetic of algebraic tori, Ann. of Math., 74 (1961), 101-139.

[8] - On the Tamagawa number of algebraic tori, Ann. of Math., 78 (1963), 47-73.

[9] M. Rosen, Representations of twisted group rings, Thesis at Princeton Univ., 1963.

[10] R. G. Swan, Projective modules over group rings and maximal orders, Ann. of Math., 76 (1962), 55-61.

[11] - The Grothendieck ring of a finite group, Topology, 2 (1963), 85-110.

[12] - Invariant rational functions and a problem of Steenrod, Invent. Math., 7 (1969), 148-158.

[13] V. E. Voskresenskiri, Birational properties of linear algebraic groups, Izv. Akad. Nauk. SSSR, 34 (1970), 3-19.

Tokyo Metropolitan University

Osaka City University 